\title{
Uniform carbon spheres of high purity prepared on kaolin by CCVD
}

\author{
Jian-Ying Miao ${ }^{\mathrm{a}}$, Dennis W. Hwang ${ }^{\mathrm{b}}$, Che-Chen Chang ${ }^{\mathrm{b}}$, Sheng-Hsien Lin ${ }^{\mathrm{a}, \mathrm{b}}$, K.V. Narasimhulu ${ }^{\mathrm{a}}$, \\ Lian-Pin Hwang a,b, * \\ ${ }^{\mathrm{a}}$ Institute of Atomic and Molecular Sciences, Academia Sinica, Taipei 106, Taiwan, ROC \\ ${ }^{\mathrm{b}}$ Department of Chemistry, National Taiwan University, Taipei, Taiwan, ROC
}

Received 17 May 2002; received in revised form 10 March 2003; accepted 11 March 2003

\begin{abstract}
Carbon spheres with diameters of 400-2000 nm were synthesized using transition metal (TM) supported on kaolin catalysts by CCVD in large quantities. The synthetic reaction was carried out at temperatures higher than $650{ }^{\circ} \mathrm{C}$. The as-synthesized carbon spheres can be purified and separated by the flow of $\mathrm{N}_{2}$ and $\mathrm{C}_{2} \mathrm{H}_{2}$ gases at 200 and 50 sccm, respectively. The carbon spheres are composed of unclosed graphene layers with interlayer distances of $0.33-0.35 \mathrm{~nm}$ between the layers. The carbon spheres were found to be thermally stable in air and no endothermic or exothermic phenomena were found in $\mathrm{N}_{2}$ atmosphere at temperatures lower than $500{ }^{\circ} \mathrm{C}$.
\end{abstract}

(C) 2003 Elsevier Science B.V. All rights reserved.

Keywords: Synthesis; Carbon spheres; Catalytic process; Catalytic chemical vapor deposition (CCVD)

\section{Introduction}

Not much attention was paid to work on carbon materials soon after the discovery of carbon onions [1] and fullerenes [2] in the 1980s. When Iijima [3] used high-resolution transmission electron microscopy (HRTEM) to observe fullerenes in 1991, sudden interest developed in many structural forms of carbon. From that time, scientists have synthesized and increasingly studied these forms of carbon, such as fullerenes and its family $\mathrm{C}_{n}$, as well as carbon nanotubes (CNTs), nanofibers, onions, trees and spheres [4-11].

As a globular form of carbon, carbon spheres have different structures from the $\mathrm{C}_{60}$ cage of carbon onions. Fullerenes represent a unique category of cage molecules with a wide range of sizes, shapes and molecular weights. In other words, fullerenes are spheroidal carbon shells and carbon onions are composed of fullerene cages with closed carbon shells.

Up to now, studies on carbon spheres have been fewer compared to other forms of carbon, such as CNTs, fullerenes, etc. In fact, carbon spheres have excellent

\footnotetext{
*Corresponding author. Tel.: +886-2-23668287; fax: +886-223620200.

E-mail address: nmra@po.iams.sinica.edu.tw (L.-P. Hwang).
}

properties similar to those of fullerenes. Carbon spheres have interesting applications in the preparation of diamond films, lubricating materials, special rubber additives, etc. However, there is an emerging need for the production of carbon spheres in large quantities under mild experimental conditions for cost-effective production.

Carbon spheres composed of microcrystalline graphite layers were reported to be synthesized by a method using catalysts of transition and/or rare earth metal oxides of mixed valence at $1100{ }^{\circ} \mathrm{C}$ [11]. Carbon spheres synthesized by this method have an average diameter of $\sim 210 \mathrm{~nm}$. Carbon spheres with a size of $1-10 \mu \mathrm{m}$ were found in carbon black produced by carbonization of polyethylene-poly(vinylchloride) in a sealed gold tube under a pressure of $30 \mathrm{MPa}$ [12], and in carbon vapor produced by decomposition of $\beta$-SiC powder [13]. Without the use of the catalysts, carbon spheres with a size of $20-500 \mu \mathrm{m}$ were formed as a side product during fullerene synthesis in flowing helium (33 mbar) at temperatures of $2500-2600{ }^{\circ} \mathrm{C}$, by depositing carbon from the gas phase [14]. Expensive set-up or hazardous experimental conditions, such as high reaction temperature and high pressure, are necessary for the above methods. This paper describes a new method of 


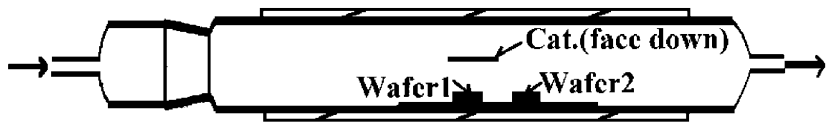

Fig. 1. Experimental set-up for synthesis of carbon spheres.

continuous preparation, purification and automatic isolation of carbon spheres. The catalytic synthesis was carried out with a new catalyst prepared with transition metal ions supported on kaolin $(\mathrm{M}=\mathrm{Fe}, \mathrm{Co}, \mathrm{Ni}, \mathrm{Cu})$ with low cost on a large scale. This catalyst is found to be highly efficient for the production of carbon spheres at relatively lower reaction temperatures than those reported in the literature.

\section{Experimental}

Transition metal $(\mathrm{M}=\mathrm{Co}, \mathrm{Ni}, \mathrm{Fe}, \mathrm{Cu})$ supported on kaolin (aluminum silicate, hydrated) was prepared by the impregnation method. Kaolin powder was sieved to $<100$ mesh. The weight ratio of kaolin to transition metal salts was approximately 1:1 in the mixture. The resulting slurry was stirred vigorously for approximately $10 \mathrm{~min}$, and then coated onto the surface of a kaolin or ceramic plate. The catalyst was then dried at $60{ }^{\circ} \mathrm{C}$ overnight in air to form a solid layer. The plate coated with the catalyst was placed on a long ceramic boat, face down. The catalyst plate and the ceramic collection boat were put into the central part of a quartz tube in a column furnace. $\mathrm{N}_{2}$ gas was used as the carrier gas at $200 \mathrm{sccm}$ during synthesis. The precursor gas $\mathrm{C}_{2} \mathrm{H}_{2}$ was flown at a rate of $50 \mathrm{sccm}$ during the reaction. The furnace was kept isothermal at a certain temperature higher than $650{ }^{\circ} \mathrm{C}$ for more than $30 \mathrm{~min}$. After the reaction, $\mathrm{N}_{2}$ was kept flowing continuously through the quartz tube until the furnace had cooled down to temperatures lower than $250{ }^{\circ} \mathrm{C}$. Uniform carbon spheres of different sizes with high purity could be collected from different parts of the collecting ceramic boat. Fig. 1 shows the experimental set-up of the reaction chamber used for the synthesis of carbon spheres.

\section{Results and discussion}

The carbon spheres collected from different parts of the ceramic boat have different sizes. The dimensions of carbon spheres obtained from identical locations are approximately the same. In this experiment, two pieces of silicon wafers were placed in different parts of the collection boat to examine the size distribution of spheres produced. One was placed underneath and the other was downstream of the catalyst plate. The final products on the surface of the silicon wafer were investigated using scanning electron spectroscopy (SEM) with a VG Microlab 350 system. The product spheres were uniform, as viewed from the SEM images. Fig. 2a,b exhibit uniform spheres of different sizes obtained on the surfaces of wafers 1 and 2, respectively. The diameter of the spheres on wafers 1 and 2 is approximately $1.8 \mu \mathrm{m}$ and $600 \mathrm{~nm}$, respectively.

Auger electron energy spectra of fresh spheres were obtained using a VG Microlab 350 system. The spectrum exhibits a peak at $\mathrm{KE}=275 \mathrm{eV}$, which coincides with the Auger electron energy of carbon. Elemental analysis of the spheres by a Philips XL-30 SEM equipped with EDS gave two peaks, as shown in Fig. 3. This shows that the carbon spheres are composed of $81 \%$ carbon and $19 \%$ oxygen, as listed in Table 1 . The spheres are made of pure carbon and no other elements were found. The presence of low oxygen concentrations may be due to absorption by the carbon spheres during storage. The fine structure of the carbon spheres was examined with a JEOL JEM-4000EX high-resolution transmission electron microscope (Fig. 4). The inset in the figure shows the microstructure of the sphere. As shown in the figure, the carbon sphere has a solid center. Graphene layers of
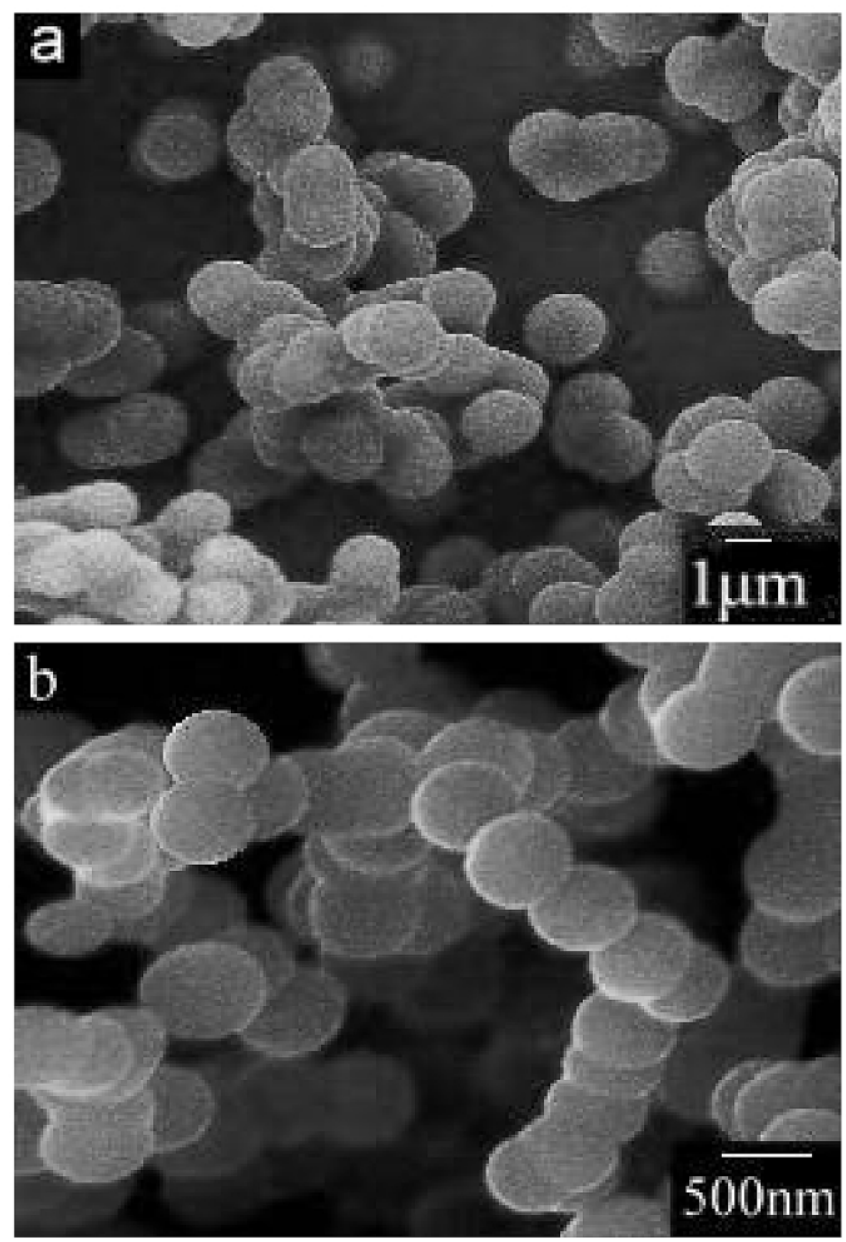

Fig. 2. SEM images of the carbon spheres synthesized with $\mathrm{Co}^{2+} /$ kaolin (a) on wafer $1(\sim 1.8 \mu \mathrm{m})$ and (b) on wafer $2(\sim 600$ $\mathrm{nm})$. 


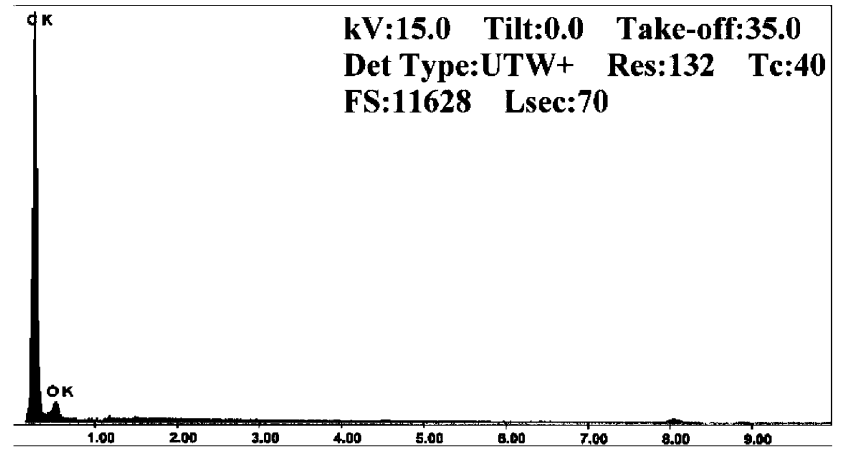

Fig. 3. EDS spectrum of the carbon spheres.

the carbon spheres have an interlayer distance of approximately $0.3-0.35 \mathrm{~nm}$.

Carbon spheres with diameters of $\sim 400$ and $\sim 600$ $\mathrm{nm}$ were investigated by thermogravimetric analysis (TGA) and differential scanning calorimetry (DSC). The TGA test was carried out using a Dynamic TGA 2950 in air and DSC using an LT-Modulate DSC 2920 in $\mathrm{N}_{2}$ with a heating rate of $3{ }^{\circ} \mathrm{C} / \mathrm{min}$. The flow rates of air and $\mathrm{N}_{2}$ were 60 and $50 \mathrm{ml} / \mathrm{min}$, respectively. The weight loss and its first derivative with respect to the sample temperature are shown in Fig. 5. The first derivative of weight loss for separated carbon spheres shows a single sharp peak in a narrow temperature range. This suggests that the carbon spheres are uniform in size. The TGA peaks for carbon spheres of different sizes show different heat-resistant abilities. Carbon spheres with a diameter of $600 \mathrm{~nm}$ remained at $90 \%$ of their weight at $551.92{ }^{\circ} \mathrm{C}$, and had the highest decomposition rate of $1.320 \% /{ }^{\circ} \mathrm{C}$ at $588.92{ }^{\circ} \mathrm{C}$. As for the smaller carbon spheres of $400 \mathrm{~nm}$ in size, $90 \%$ of the sample was left at $514.17^{\circ} \mathrm{C}$ and decomposed at 555.16 ${ }^{\circ} \mathrm{C}$ at a fast rate of $1.635 \% /{ }^{\circ} \mathrm{C}$. DSC results showed no endothermic or exothermic phenomena at temperatures lower than $500{ }^{\circ} \mathrm{C}$. This implies that the carbon spheres are thermally stable up to $500{ }^{\circ} \mathrm{C}$.

The continuous process of synthesis, purification and isolation of the carbon spheres was achieved via the continuous flow of gases at controlled reaction temperatures. Intermediate carbon materials form along the carbon spheres on the surface of the catalyst with kaolin as the template, which eliminates dangling bonds and reduces the surface tension. Molecular dynamics studies

Table 1

Composition of carbon spheres obtained from EDS analysis

\begin{tabular}{lrrrrrr}
\hline Element & \multicolumn{2}{l}{ Composition } & \multirow{2}{l}{$K$-ratio } & $Z$ & $A$ & \multicolumn{1}{l}{$F$} \\
\cline { 2 - 4 } & (wt.\%) & (at.\%) & & & & \\
\hline C K & 80.98 & 85.01 & 0.6740 & 1.0035 & 0.8293 & 1.0001 \\
O K & 19.02 & 14.99 & 0.0404 & 0.9848 & 0.2158 & 1.0000 \\
Total & 100.00 & 100.00 & & & & \\
\hline
\end{tabular}

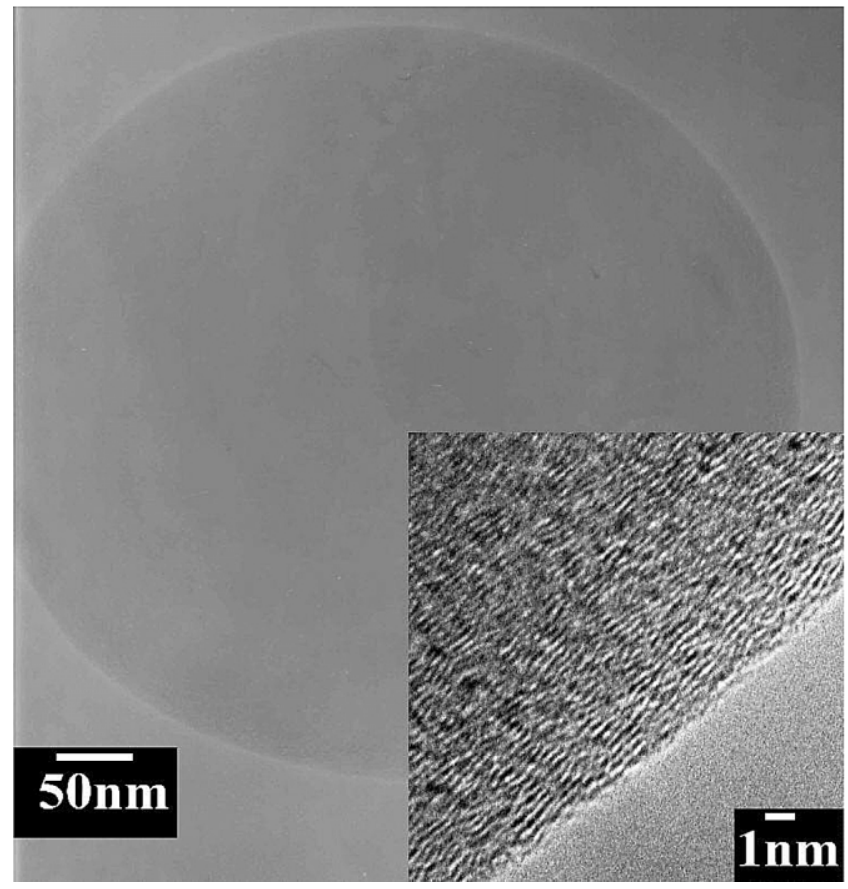

Fig. 4. High-resolution TEM image of a carbon sphere (insert shows the microstructure of the sphere).

by Brabec et al. [15] of the growth of carbon nanotubes have shown that it is the development of pentagonal rings that gives rise to a curved geometry at the tube tip and leads to tube closure. During the closure, hexagonal structures, which develop in the straight helical tube of carbon nanotube, and then heptagon rings, may become unstable, and those at step edges may quickly degenerate into pentagon pairs upon annealing. Results from ab initio studies [16] also showed that closure of the tubes depends on the local stability of the accessible atomic configuration. Experimental observations in the present work indicate that our growth

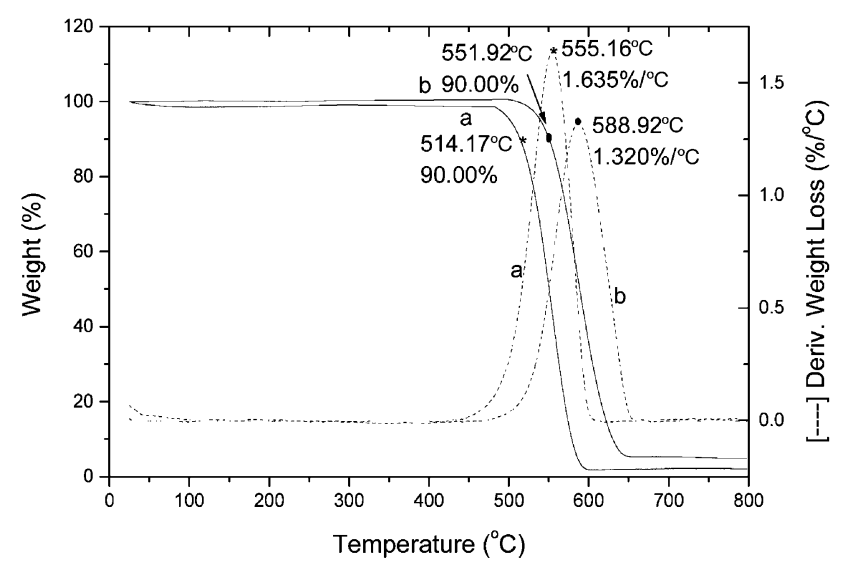

Fig. 5. TGA scan (weight loss and the first derivative with respect to temperature) in air for the carbon spheres. 


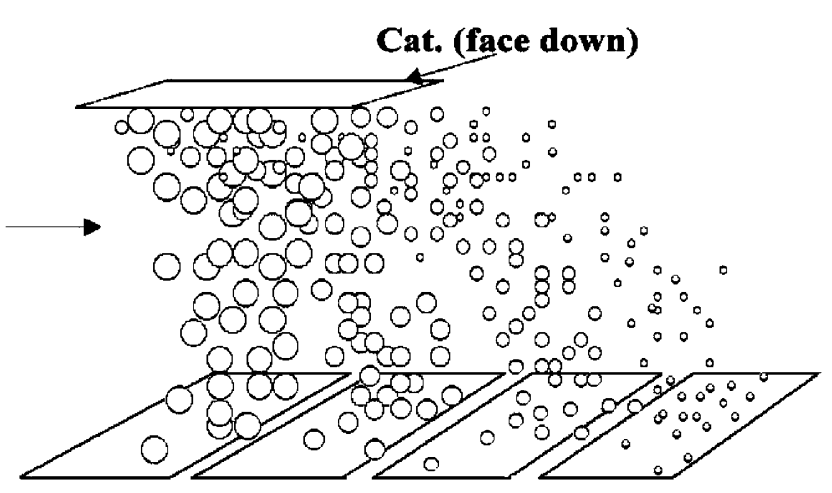

Fig. 6. The entire process of preparation, purification and isolation of carbon spheres.

conditions do not favor the formation of stable hexagonal or heptagonal structures. The intermediate carbon materials produced in the presence of the catalyst used may contain unstable hexagons or heptagons or reactive dangling bonds. Bond switching and migration processes [15] may then take place, which lead to the absence of straight tubes, and thus the formation of spherical carbon structures occurs. The detailed formation mechanism for carbon spheres with this method will be the focus of other work by our group under a different topic. In these experiments, the flow of carrier gas provides a means of isolating carbon spheres of different sizes. Fig. 6 demonstrates the whole procedure in a single step.

The final carbon spheres dropped out on different parts of the collection boat, depending on their weight. An increasing number of carbon spheres of uniform size piled up on the surface of the boat. An approximate thickness of $2 \mathrm{~mm}$ of carbon spheres was obtained while the reaction was carried out at $\sim 700{ }^{\circ} \mathrm{C}$ for $1 \mathrm{~h}$, with flow rates of $\mathrm{N}_{2}$ and $\mathrm{C}_{2} \mathrm{H}_{2}$ at 200 and $50 \mathrm{sccm}$, respectively. SEM images in Fig. 7 exhibit the piling up process for carbon spheres on the surface of a silicon wafer. The arrow in Fig. 7d indicates direction of the piling up of carbon spheres on the surface of silicon wafer. The whole process involves low-cost synthesis of carbon spheres in large quantities, as well as purification and isolation of the final product. The flow rates of the
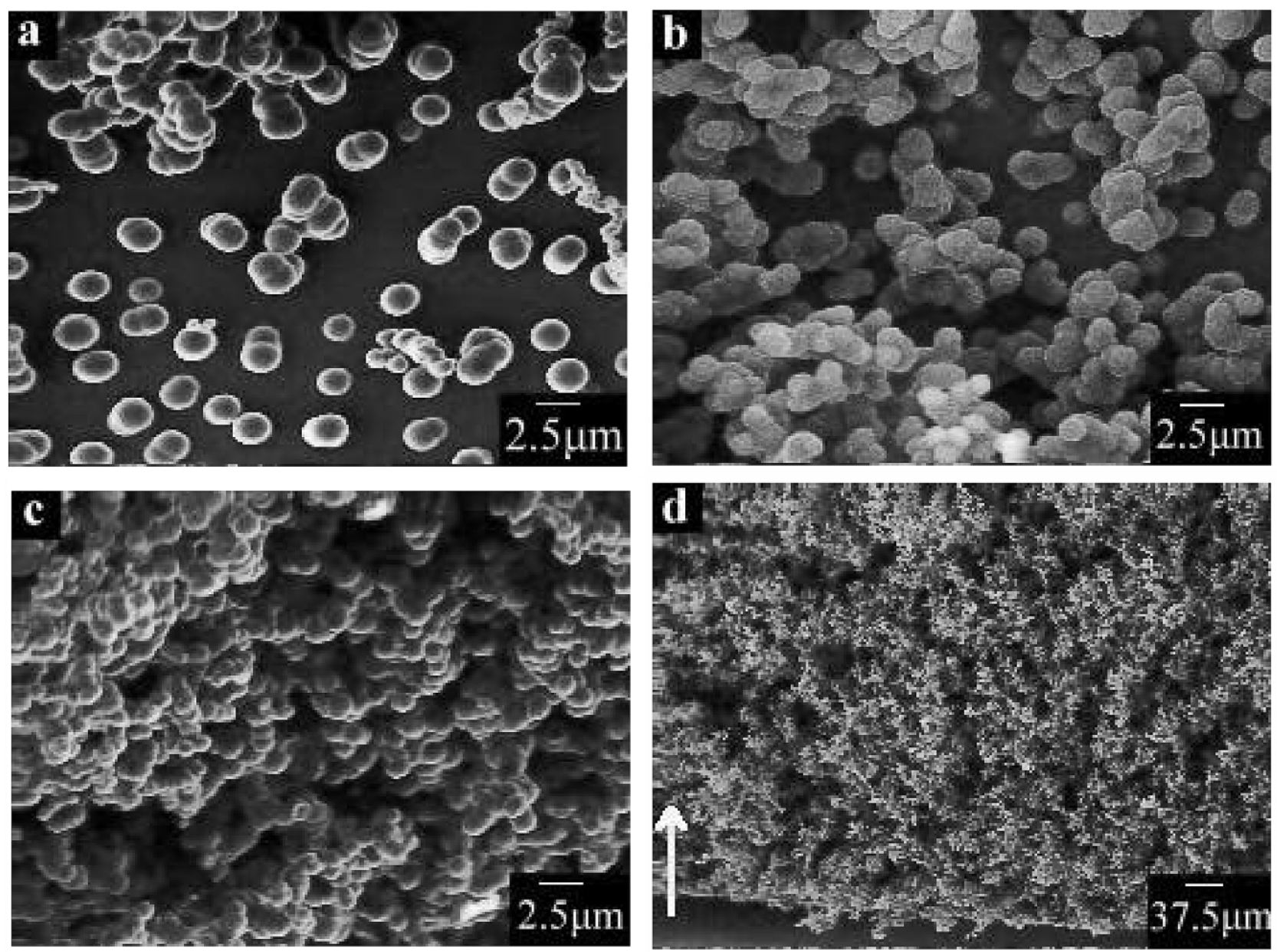

Fig. 7. SEM images of the piling up process: $(\mathrm{a}-\mathrm{c})$ the piling up process; and (d) carbon spheres piled up along the direction of the arrow on the surface of the silicon wafer. 
carrier gas and the precursor play an important role in the purification and isolation processes.

\section{Conclusions}

The tailored synthesis of carbon spheres of different sizes in large quantities was successfully carried out using a template method in CCVD with TM ( $\mathrm{Fe}$, Co, $\mathrm{Ni}, \mathrm{Cu}$, etc.) supported kaolin catalysts. We explained the use of the catalyst in the CVD technique to synthesize the carbon spheres. We have also demonstrated that the use of a TM-supported kaolin catalyst enables the entire process of preparation, purification and isolation of pure carbon spheres to be carried out in the reaction chamber in a one-step reaction at mild temperatures just above $650{ }^{\circ} \mathrm{C}$.

\section{Acknowledgments}

This work was financially supported by the CTCI Foundation of Taiwan. All experiments were carried out on the instruments of the Instrumentation Center of the NSC of Taiwan. The financial assistance imparted by the National Science Council, ROC through grants NSC 90-2113-M-001-045 and 91-2113-M-001-037 is gratefully acknowledged.

\section{References}

[1] S. Iijima, J. Cryst. Growth 50 (1980) 675.

[2] W. Krätschmer, L.D. Lamb, K. Fostiropoulos, D.R. Huffman, Nature 347 (1990) 354.

[3] S. Iijima, Nature 354 (1991) 56.

[4] J. Hunter, J. Fye, M.F. Jarrold, Science 260 (1993) 784.

[5] Q.L. Zhang, S.C. O’Brien, J.R. Heath, et al., J. Phys. Chem. 90 (1986) 525.

[6] A.K. Sinha, D.W. Hwang, L.P. Hwang, Chem. Phys. Lett. 332 (2000) 455.

[7] G. Kamalakar, D.W. Hwang, L.P. Hwang, J. Mater. Chem. 12 (2002) 1.

[8] R.L.V. Wal, T.M. Ticich, V.E. Curtis, J. Phys. Chem. B 104 (2000) 11606.

[9] N. Sano, H. Wang, M. Chhowalla, I. Alexandrou, G.A.J. Amaratunga, Nature 414 (2001) 506.

[10] P.M. Ajayan, J.M. Nugent, R.W. Siegel, B. Wei, P. KohlerRedlich, Nature 404 (2000) 243.

[11] Z.L. Wang, Z.C. Kang, J. Phys. Chem. 100 (1996) 17725.

[12] M. Inagaki, K. Kuroda, M. Sakai, Carbon 21 (1983) 231.

[13] K. Yamada, S. Tobisawa, Carbon 27 (1989) 845.

[14] I. Loa, C. Mschel, A. Reich, W. Assenmacher, K. Syassen, M. Jansen, Phys. Status Solidi b 223 (2001) 293.

[15] C.J. Brabec, A. Maiti, C. Roland, J. Bernholc, Chem. Phys. Lett. 236 (1995) 150.

[16] A. Maiti, C.J. Brabec, C.M. Roland, J. Bernholc, Phys. Rev. Lett. 73 (1994) 2468. 